\title{
Photometric and polarimetric clues to the circumstellar environment of RY Lupi ${ }^{\star}$
}

\author{
N. Manset ${ }^{1}$, P. Bastien ${ }^{2}$, F. Ménard ${ }^{3}$, C. Bertout ${ }^{4}$, A. Le Van Suu ${ }^{5}$, and L. Boivin ${ }^{2}$ \\ ${ }^{1}$ Canada-France-Hawaii Telescope Corporation, 65-1238 Mamalahoa Hwy Kamuela HI 96743, USA \\ e-mail: manset@cfht.hawaii.edu \\ 2 Département de physique and Observatoire du Mont Mégantic, Université de Montréal, Montréal QC, Canada \\ 3 Laboratoire d'Astrophysique de Grenoble, UMR UJF-CNRS 5571, Observatoire de Grenoble, Université Joseph Fourier, BP 53 , \\ 38041 Grenoble Cedex 9, France \\ ${ }^{4}$ Institut d'Astrophysique, 98bis Bd. Arago, 75014 Paris, France \\ 5 CPPM - Centre de Physique des Particules de Marseille, CNRS/IN2P3, Université de la Méditerranée Aix-Marseille II, \\ 163 Avenue de Luminy, Case 907, 3288 Marseille Cedex 9, France \\ Received 10 September 2008 / Accepted 10 February 2009
}

ABSTRACT

\begin{abstract}
Aims. We investigate the stellar and circumstellar properties of the bright southern T Tauri star RY Lup, a G-type star showing type III variability.

Methods. We report simultaneous $B V$ polarimetric and $U B V$ photometric observations obtained during 12 consecutive nights on the $1.0 \mathrm{~m}$ and $50 \mathrm{~cm}$ telescopes of the European Southern Observatory at La Silla. We compare these data to models.

Results. The polarization is high $(\approx 3.0 \%)$ when the star is faint and red $(V \approx 12.0, B-V \approx 1.3)$, and it is low $(\approx 0.5 \%)$ when it is bright and bluer $(V \approx 11.0, B-V \approx 1.1)$. The photometric and polarimetric variations share a common period of $3.75 \mathrm{~d}$. Irregular light variations, larger at shorter wavelengths, are also superposed on the cyclic variations and may be due to processes different than the one producing the periodic variations. The linear polarization is produced by dust scattering in an asymmetric (flat) circumstellar envelope. The photometric and polarimetric variations can be explained with an almost edge-on circumstellar disk that is warped close to the star, where it interacts with the star's magnetosphere. The inhomogeneous disk matter contained in the warp corotates with the star and partially occults it during part of the rotation period, which explains the dips in luminosity and the accompanying increase in polarization. All the information available on RY Lup is consistent with a system comprising a G8 star surrounded by an edge-on disk, and we find that the mass of RY Lup is $M_{\text {star }} / M_{\odot}=1.71 \pm 0.43$, while its age is $(1.2 \pm 0.4) \times 10^{7} \mathrm{yr}$.
\end{abstract}

Key words. stars: pre-main sequence - stars: variables: general - stars: circumstellar matter - polarization - methods: observational

\section{Introduction}

Solar-type young stellar objects (T Tauri stars; TTSs herafter) display light variations of up to a few magnitudes on many timescales at all wavelengths. Herbst et al. (1994) have identified three main physical processes at work in these objects: (a) magnetic activity, (b) accretion power, and (c) variable extinction caused by occultation of the stellar surface by circumstellar matter. These authors define three types of photometric variability.

Type I: Rotational modulation caused by cold magnetic spots. These are most easily detectable in the weak emission lines T Tauri stars (WTTSs). The maximum amplitude that can be attributed to cold magnetic spots is about $0.8 \mathrm{mag}$ in $V$ and $0.5 \mathrm{mag}$ in $I$.

Types II and IIp: Variability caused by cold and hot spots. Type II variations are either irregular or periodic, in which case they are called type IIp, and occur only in classical T Tauri stars (CTTSs).

Type III: Variable stellar obscuration by circumstellar dust. Also called UX Ori type variability, this type occurs in some TTSs but is mainly observed in higher mass Herbig $\mathrm{Ae} / \mathrm{Be}$ stars (HAEBESs). Type III variability is characterized

* Based on observations collected at the European Southern Observatory in La Silla, Chile. by large amplitude light variations in stars with no evident veiling or effective temperature variations. Type III variables often fluctuate around their high-luminosity state, while sometimes fading suddenly by several magnitudes before recovering their previous luminosity on a timescale of days, and the fading episodes are often cyclic. That the degree of linear polarization increases as the star becomes faint is a major argument supporting the idea that variable obscuration is responsible for the stars fading. For more details, see Ménard \& Bertout (1999).

Type III variability is observed among a sizable fraction of HAEBEs, suggesting that disk flaring and/or the puffed up innermost rim of the disk may play a role in occulting the star even when the disk is seen at medium-high inclination angles; cf. Isella \& Natta (2005) and references therein. Its rarer occurrence in late CTTSs suggests a different structure of the inner disk, which may be warped in the vicinity of the magnetospheric radius (e.g., Bouvier et al. 2003). A study of type III variability in intermediate mass objects may thus shed light on the physical processes that are responsible for the different inner disk structures shown by objects of various stellar masses. This objective provides an incentive for the present investigation of the RY Lup simultaneous photometric and polarimetric variability.

The TTS RY Lupi (=HBC $252=$ HIP 78317) is located in the Lupus $\mathrm{T}$ association at $l=337.35, b=+9^{\circ} .62$. The marginally significant Hipparcos parallax of $9.26 \pm 2.83$ mas 
(Perryman et al. 1997) indicates a distance of $108_{-25}^{+48} \mathrm{pc}$. The distance estimated by Bertout et al. (1999) for the Lupus 1, 3, and 4 clouds $\left(147_{-27}^{+42} \mathrm{pc}\right)$ includes the individual parallax of RY Lupi but also better constrained values for a few other stars, and therefore appears somewhat more reliable. It is also more in line with previously derived distance estimates for that starforming region (see for example Alves \& Franco 2006; and Franco 2002).

There are several estimates of RY Lup's spectral type, which was determined as K1:ea V (Li) by Herbig (1977) and as K4 by Appenzeller et al. (1983). Based on the latter spectral type and their own photometric data, Hughes et al. (1994) deduced a mass range $0.94 \leq M / M_{\odot} \leq 1.19$ and and an age range $1.6 \times 10^{6} \leq t(\mathrm{yr}) \leq 3.2 \times 10^{6}$ for the star. From an extended set of spectroscopic data obtained over several years, Gahm et al. (1989) concluded that the spectral type of RY Lup is G8 IV-V with an uncertainty of one spectral subclass. They also found that the type does not change as the star becomes fainter. In contrast to many CTTSs with rich emission-line spectra, line emission in RY Lup is usually restricted to $\mathrm{H} \alpha$, and sometimes this line is not even seen in emission (Appenzeller et al. 1983). However, the $\mathrm{H} \beta$ line goes in emission when the star gets fainter (Evans et al. 1982; Liseau et al. 1987; Hutchinson et al. 1989).

The spectral energy distribution of RY Lup shows a sizable infrared excess, as well as modest UV excess (Evans et al. 1982; Gahm et al. 1989). The photometric variability of the star was discovered by Woods on Harvard plates in 1921 (Hoffmeister 1958), and the star was classified as a typical RW Aur star on the basis of its lightcurve (Hoffmeister 1949). From a long-term study of the optical photometry of the star, Gahm et al. (1989) report (a) significant photometric variations on timescales of hours, (b) a constant variability with a $3.75 \mathrm{~d}$ period over $\approx 52$ years, and (c) a decline in the mean and maximum photometric magnitudes over decades. Other investigators derived slightly different periods for the photometric variability: $3.6 \mathrm{~d}$ (Boesgaard 1984; Hutchinson et al. 1989), 3.67 d (Hoffmeister 1958), and $3.9 \mathrm{~d}$ (Bouvier et al. 1986).

The photometric behavior of RY Lup is typical of type III variability. The star gets redder as it gets fainter (Evans et al. 1982; Liseau et al. 1987; Hutchinson et al. 1989; Covino et al. 1992), and the amplitude of the variations also decreases with wavelength. Furthermore, Liseau et al. (1987) and Gahm et al. (1989) report color flips in $B-V$, where the color changes by one magnitude in less than $20 \mathrm{~h}$ from an extremely blue to an extremely red color. The infrared photometric properties of RY Lup were investigated by Glass \& Penston (1974), who report a lightcurve of type I0.6s and an emission class I, based on Herbig \& Rao's (1972) notation.

The intrinsic position angle of T-Tauri-star polarization is generally a function of both wavelength and time, i.e., $\theta_{\star}=$ $\theta_{\star}(\lambda, t)$ (Bastien 1981); see also Bastien (1988a) for a review of young-stellar-object polarization properties, and RY Lup is a good example of this behavior (Bastien 1985). These authors also noticed remarkably large and rapid variations in both polarization and position angle in RY Lup. Drissen et al. (1989) have confirmed RY Lup's variable polarization. Their data did not show any periodicity but displayed a correlation in the sense that the polarization is higher when the star is fainter.

Bastien (1982, 1985, 1988a) compiled all the polarimetric and photometric (both visual and photoelectric) data available in the literature on $\mathrm{T}$ Tauri stars and did not find any correlation between the polarization, $U B V R I$ photometry, and equivalent widths of emission lines. The polarization is, however, well-correlated with IR photometric data. Simultaneous photometry and polarimetry of RY Lup was reported by
Hutchinson et al. (1994), who find that the polarization increases as the star gets redder and fainter, and $\theta$ also changes. Finally, we note that no circular polarization was seen by Hutchinson et al. (1994), although Yudin \& Evans (1998) detected it at the $3 \sigma$ level.

The paper is organized as follows. We present our observations in Sect. 2, followed by an analysis of the photometric and polarimetric data in Sect. 3. A discussion of possible mechanisms for the explanation of the photometric and polarimetric variations is presented together with a polarimetric model and the derived properties of RY Lup in Sect. 4. Our conclusions follow.

\section{Observations}

Simultaneous photometric and polarimetric observations were carried out on the $50 \mathrm{~cm}$ and $1.0 \mathrm{~m}$ telescopes, respectively, of the European Southern Observatory (ESO) at La Silla from 1982 February 12 to 24, using the ESO photometer and polarimeter ${ }^{1}$. Standard $U B V$ filters were used for the photometric observations. The polarimetric bandpasses approximate the $B V$ bands and are defined by the following central wavelengths and bandwidths: $4300 \AA$ (1000 $\mathrm{\AA})$ and $5250 \AA$ (500 $)$. Data were reduced with the standard ESO data reduction programs.

During the same observing period, we also observed every night ${ }^{2}$ primary photometric standard stars and two comparison stars within $1^{\circ}$ from RY Lup, HD 14224 and HD 14262. The photometric data for RY Lup are listed in Table 6. In the following, we also make use of $U B V R I$ data obtained with the same ESO $50 \mathrm{~cm}$ telescope in 1983 June-August by Bouvier et al. (1988).

Concerning the polarimetric observations, standard polarized and unpolarized stars were observed throughout the observing run. The instrumental polarization was estimated to be $<0.05 \%$, and was therefore neglected. The polarization data are given in Tables 7 and 8, where one finds the Julian date JD-2 400 000.0, the polarization and its error, the equatorial position angle and its error, and the phase (see below) in Col. 1 through 6.

The photometric and polarimetric data obtained over the 12 consecutive nights are presented as a function of the Julian date in Fig. 1. Large-amplitude variations in both photometric and polarimetric data are clear. These amplitudes are typically $1.2,0.25$, and $0.37 \mathrm{mag}$, in $V, B-V$, and $U-B$, respectively, and $2.3 \%, 48^{\circ}, 1.8 \%, 47^{\circ}$, for the polarization level and positions angle at $4300 \AA$ and $5250 \AA$, respectively. The extreme values are even higher. However, the most striking feature of the data is that, when the star is bright the polarization is low and when the polarization is high, the star is fainter and also redder.

\section{Analysis of observations}

\subsection{Period search}

A period was searched for in our data sets using a very simple sinusoid fit. Periods between 0.1 and 12 days were considered. All data were taken into account when looking for a period: the polarization and position angles at $4300 \AA$ and $5250 \AA$, as well as the $U B V$ and $U B V R I$ data. The 1982 and 1983 photometric

\footnotetext{
1 The instruments were decommissionned long ago. Information and technical specifications are available directly from ESO upon request. See http://www.eso.org/sci/facilities/lasilla/ instruments / for more details.

2 Except 1982 February 14-15 (UT).
} 


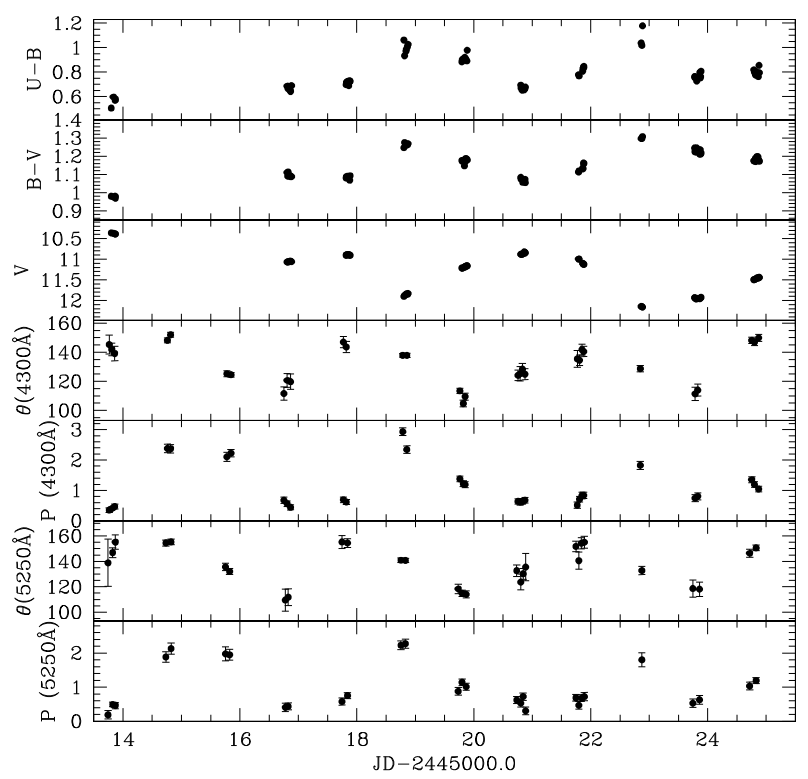

Fig. 1. Photometry and polarimetry (observed polarization, i.e. intrinsic + interstellar) of RY Lup at $4300 \AA$ and $5250 \AA$ as a function of the Julian date.

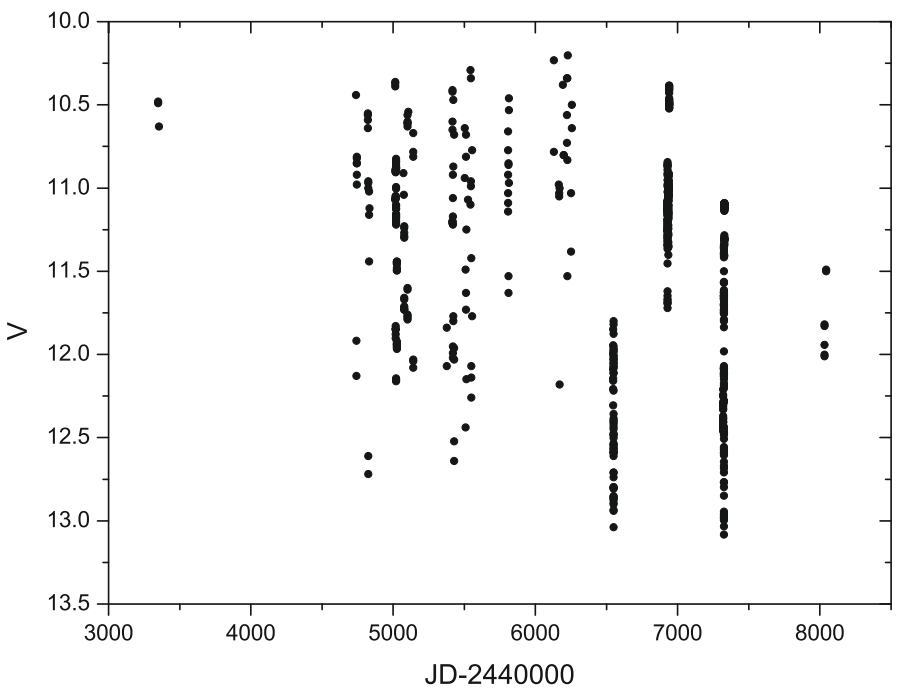

Fig. 2. Observed $V$ magnitudes of RY Lupi from 1977 to 1990.

data were searched independently; i.e., it was not assumed that the phase was conserved from 1982 to 1983.

We derived an average period of $3.74_{-0.18}^{+0.20}$ days. The errors indicated are the standard deviations of the best frequencies. This value is in agreement with the value of 3.75 days found by Gahm et al. (1989) from a compilation of data for the past 52 years. It also agrees with the value derived above for the photometric observations ranging from 1977 to 1990.

To compare our observations to the photometric variability of RY Lupi on longer timescales, we added to our own measurements the optical photometric data gathered by Gahm et al. (1993a) and Herbst et al. (1994) for the period extending from the late 1970s to 1990. Figure 2 displays the 622 $V$-measurements contained in the database and Fig. 3 shows how they are distributed in half-magnitude intervals. There is less than a $10 \%$ chance of observing RY Lupi within 0.5 mag of its brightest state in $V$, but a $60 \%$ chance of observing it in a state brighter than $V=11.5 \mathrm{mag}$. The star is fainter than $V=$ 12.5 about $10 \%$ of the time. The data shown in Fig. 2 present

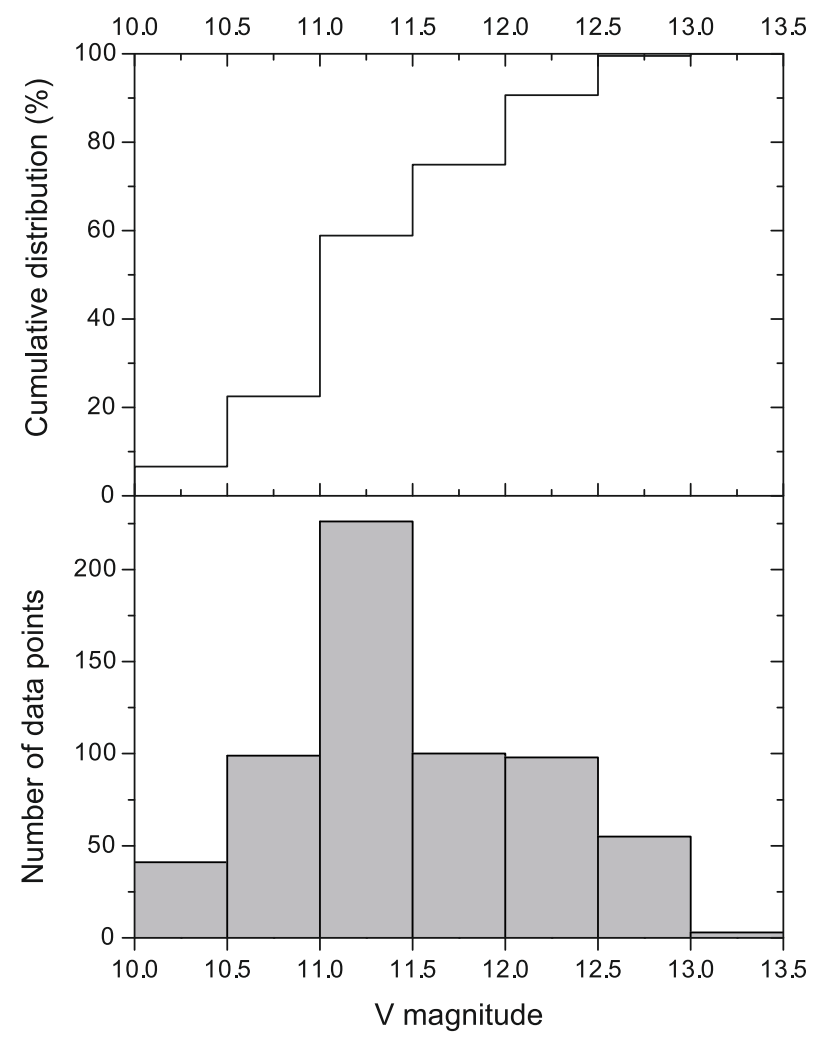

Fig. 3. Bottom panel: histogram of observed $V$ magnitudes from 1977 to 1990. Top panel: the cumulative distribution of $V$ observations.

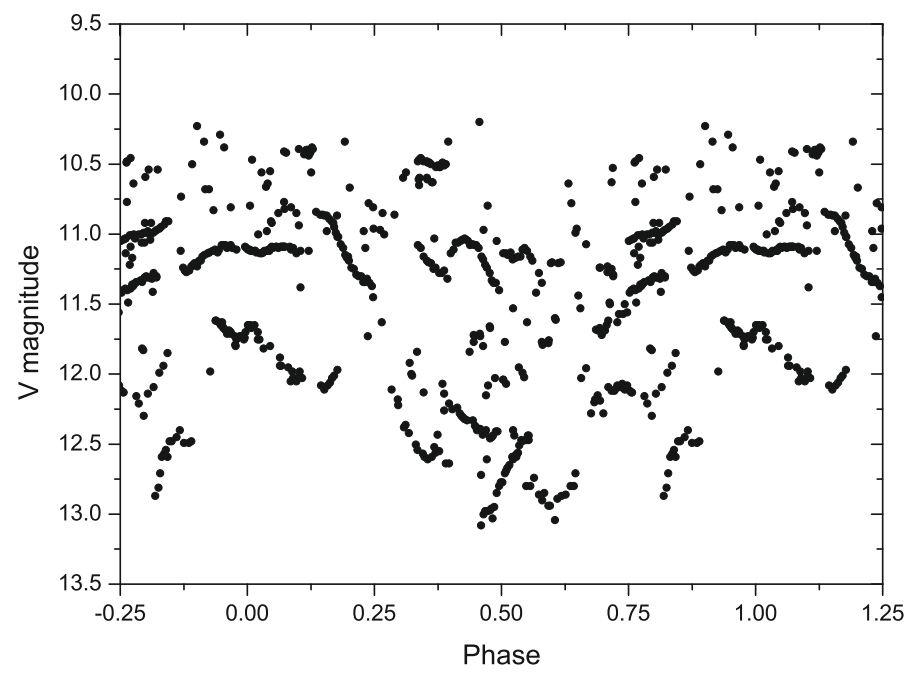

Fig. 4. Same data as in Fig. 2, but phase-folded with a 3.75 d period.

cyclical variations with a period of $3.75 \mathrm{~d}$, based on a CLEANed periodogram analysis (Baisch \& Bokelmann 1999) of the $V$ data and Monte Carlo simulations showing that the probability for the derived period to be due to chance is less than $0.1 \%$. No other peak in the periodogram reaches this significance level. Figure 4 shows that the derived period does not account for the full variability of the star. Obviously, there are additional, random light variations that complicate the photometric behavior of RY Lup.

The 3.75 d-period was used, along with an arbitrary epoch of JD $=2445000.0$, to compute the phase diagrams presented in the rest of this paper. The photometric and polarimetric data are presented together as a function of the orbital phase in Fig. 5. 


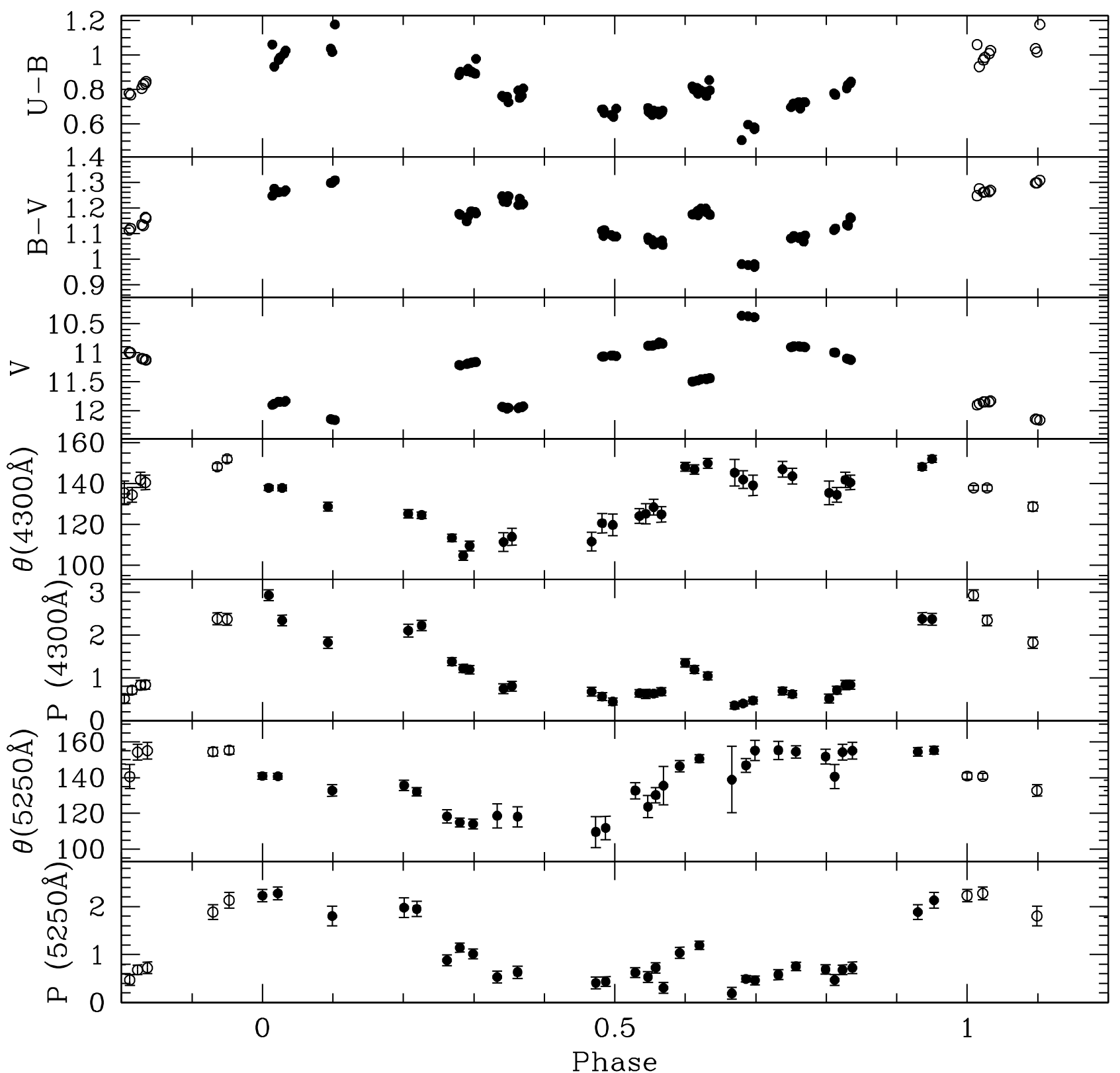

Fig. 5. Photometry and polarimetry (observed polarization, i.e. intrinsic + interstellar) of RY Lup at $4300 \AA$ and $5250 \AA$ as a function of phase, calculated with a period of $3.75 \mathrm{~d}$. The open circles are data points repeated for clarity.

\subsection{Photometric observations}

The color-magnitude diagrams of the photometric data are shown in Fig. 6. It is immediately obvious that RY Lup gets redder as it gets fainter as noted by previous investigators. The so-called blueing effect (i.e., when a star goes from getting redder to getting bluer as it continues to get fainter; cf. Evans et al. 1982, Hutchinson et al. 1987, Gahm et al. 1993b) is not seen in our data, but this is hardly surprising since this effect has only been observed in RY Lup for $V$ magnitudes fainter than about 12.0, a magnitude that our data barely reach.

The color-magnitude graphs also show that the $B-V$ vs. $V$ relationship is much tighter than the $U-B$ vs. $V$ one; there is more scatter in the $U-B$ color as the star gets fainter. The $B-V$ vs. $V$ diagram has a slope of 6.1. The $U-B$ vs. $V$ upper envelope has a slope of 7.2, whereas the lower envelope has a slope of 2.1. Variations are not monotonic, and go back and forth.
The photometry does not show systematic trends as a function of time. As the $V$ magnitude goes up or down, colors can go either way. One interesting feature is that for the 2 photometric minima, there is more scatter in $U-B$, but not in $B-V$, but that is in part due to the low accuracy of $U$ measurements when the star is faint.

\subsection{Polarimetric observations}

Generally, polarimetric observations include both intrinsic polarization produced at the target and interstellar (IS) polarization. The Heiles (2000) polarization catalog, which contains polarimetric observations for more than 9000 stars, was used to estimate the interstellar polarization in the vicinity of RY Lup by selecting 6 stars located within $35 \mathrm{pc}$ and $15^{\circ}$ of it. We assumed that RY Lup's distance is $150 \mathrm{pc}$ (Bertout et al. 1999) and that the visual extinction is $A_{\mathrm{V}}=0.47$ (see our calculation of $A_{\mathrm{V}}$ in 

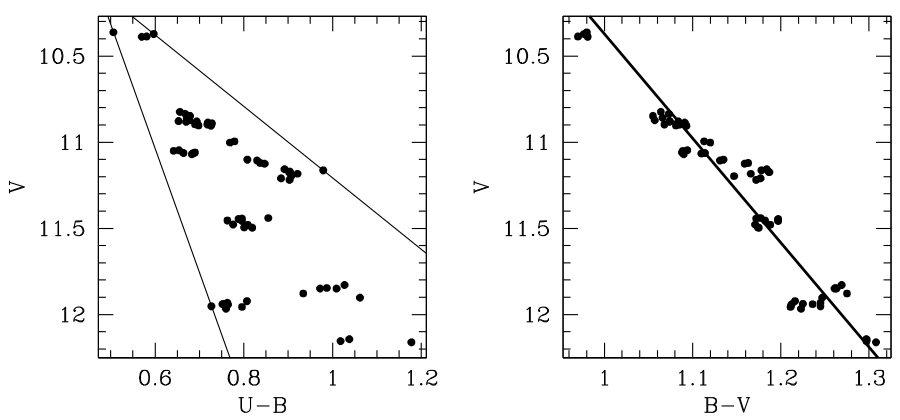

Fig. 6. $V$ magnitude as a function of $U-B$ and $B-V$ colors.

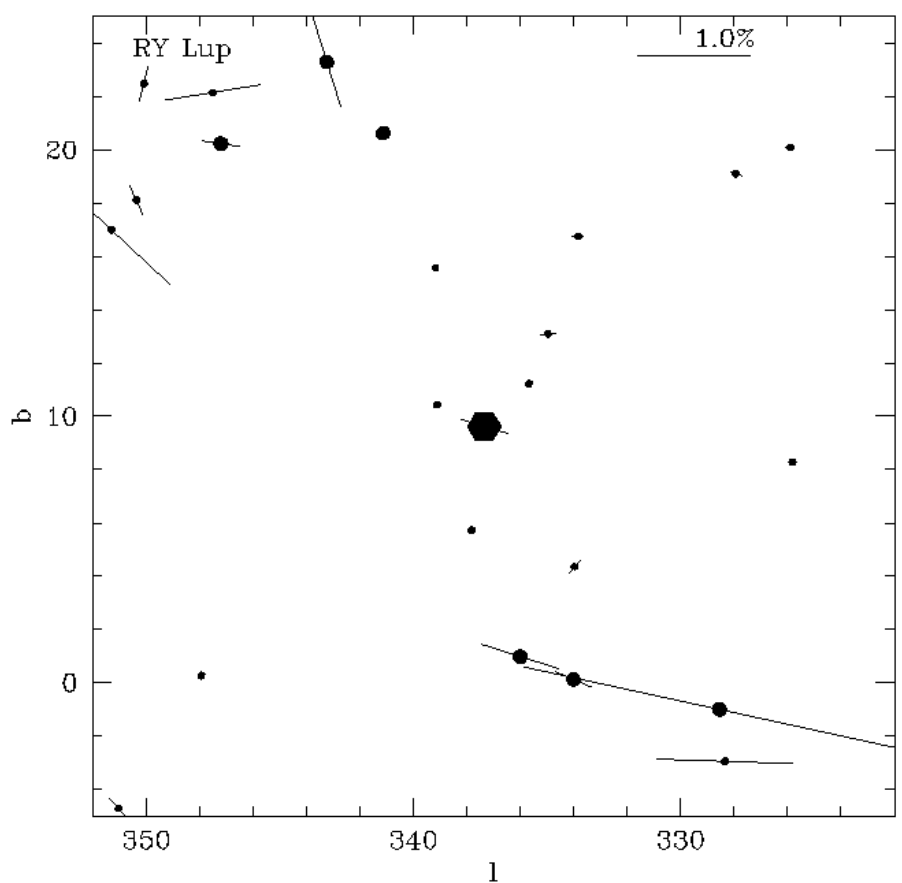

Fig. 7. Map of the interstellar polarization in the vicinity of RY Lup. The computed average interstellar polarization for RY Lup is shown at the position of the hexagon. Stars used to determine the average IS polarization are drawn with larger symbols than other stars, which are either outside the $15^{\circ}$ circle, have a value of $E(B-V)$ in the Heiles catalog $\leqslant 0.0$, or yield a ratio of $P / E(B-V)>9.0$.

Sect. 4). The distance interval chosen, $35 \mathrm{pc}$, comes from the average of the error bars ${ }_{-27}^{+42}$ on the distance found by Bertout et al. (1999). The map of IS polarization is presented in Fig. 7. The IS distance-weighted polarization calculated by averaging the polarization of the 6 stars is $0.44 \% \pm 0.20 \%$ in the $V$ band, with a distance-weighted equatorial average position angle of $30 \pm 9^{\circ}$. See Manset \& Bastien (2002) for more details about our averaging procedure.

If one chooses different distances or distance intervals, e.g., $110 \pm 35$ pc (8 stars), $120 \pm 35$ pc ( 9 stars $), 150 \pm 35$ pc (6 stars), and $150 \pm 75 \mathrm{pc}$ (14 stars), the results are similar those given above. For example, $150 \pm 75$ pc yields an interstellar polarization of $0.55 \% \pm 0.16 \%$ and at a position angle of $39 \pm 10^{\circ}$. Therefore we can conclude that the derived IS polarization is robust.

As seen in Tables 7 and 8, RY Lup's observed weighted average polarization is $0.92 \%$ at $134^{\circ}$ at $4300 \AA(N=31)$, and $0.84 \%$ at $139^{\circ}$ at $5250 \AA(N=29)$. These levels of polarization are higher than the IS polarization and indicate the presence of an intrinsic polarization component in RY Lup's measurements.
The IS position angle is also different than RY Lup's, which provides another clue for the presence of intrinsic polarization.

Using the above estimate of the IS polarization in the $V$ band and Serkowski's law for IS polarization (Serkowski et al. 1975), we derived the IS polarization value for the 2 observed wavelengths. We assumed that $\lambda_{\max }=5500 \AA$ for Serkowski's law (a typical value) and that the position angle of the IS polarization is constant as a function of wavelength. After subtracting this estimated IS polarization from the observed one, we found that the average values of intrinsic polarization for RY Lup were $1.30 \%$ at position angle $130^{\circ}$ at wavelength $4300 \AA$, and $1.22 \%$ at position angle $133^{\circ}$ at wavelength $5250 \AA$. RY Lup's intrinsic polarization is thus high, which suggests that RY Lup's circumstellar matter is distributed in a disk rather than in a spherical shell.

If RY Lup's disk is comparable to other CTTS disks, it is likely to be very optically thick (Ménard \& Bertout 1999). Therefore, since the intrinsic polarization is oriented in direction $\approx 130^{\circ}$ and the polarization is parallel to the scattering plane (the equatorial plane of the circumstellar disk), we predict that RY Lup's disk's equatorial plane is oriented in direction $\approx 130^{\circ}$. In contrast, an optically thin disk would be oriented at $\approx 40^{\circ}$. RY Lup has been observed extensively with WFPC2 and adaptive optics on HST, and no disk was detected (Stapelfeldt, private communication).

We also note that polarimetric variations have a higher amplitude in the blue at $4300 \AA$ than in the green at $5250 \AA$. The observed position angle varies by $\approx 45^{\circ}$ at both wavelengths. The polarization and position angle at $4300 \AA$ are correlated with the polarization and position angle at $5250 \AA$; see Fig. 8, where data obtained within $63 \mathrm{~min}$ in both filters are plotted. The correlation seen in position angles between the two filters is a consequence of the fact that the intrinsic polarization variations are significantly larger than the value of the IS polarization.

When plotting the polarimetric data in the $Q-U$ plane, one finds that our observations trace an ellipse, spending more time around $(Q, U) \approx(0.0,-0.5)$ (see Figs. 9 and 10$)$. The parameters of the fitted ellipses are given in Table 1 . The variations are periodic with a period of $3.75 \mathrm{~d}$, with second harmonics and even higher harmonics present as will be discussed in Sect. 4.2. The axis of both ellipses (Figs. 9 and 10) is compatible with the orientation of the position angle of the intrinsic polarization obtained by subtracting the IS polarization.

We finally note that polarimetric data presented by Drissen et al. (1986) are much more erratic than seen here and do not display any periodic variations, using periods of $3.75 \mathrm{~d}$ or $7.5 \mathrm{~d}$. On the other hand, the few data points presented by Bastien (1985) seem to show a maximum in polarization around phase 0.0 , when using the same epoch and period of $3.75 \mathrm{~d}$, which is compatible with the behavior reported here (see Fig. 5).

Figure 11 shows the photometric and $4300 \AA$ polarimetric data obtained within $29 \mathrm{~min}$ (0.02 JD) of one another. When RY Lup is faint, its polarization is maximum at both wavelengths, with no delay in time between them. The polarization is correlated with the magnitude and the colors. There is no correlation between the position angle and the photometric data. The $5250 \AA$ A polarimetric data show a qualitatively similar behavior.

\section{Discussion}

As briefly explained in the introduction, the main causes of light variability in TTSs are the presence of cold and/or hot spots on the stellar surface (with related episodes of magnetic flaring and 

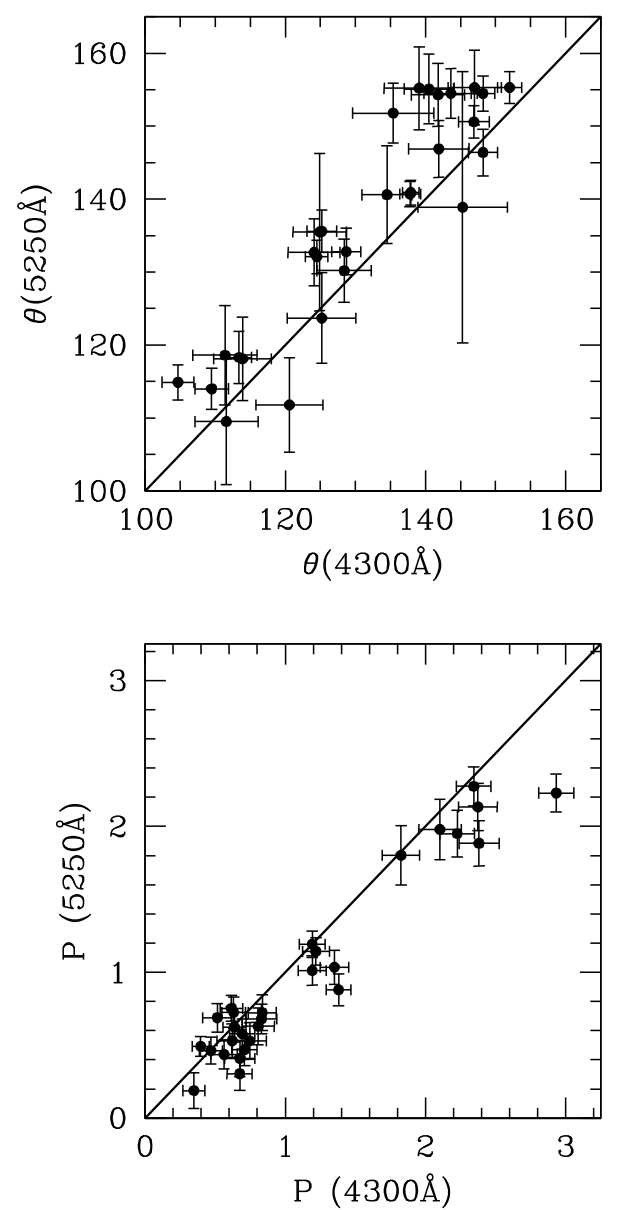

Fig. 8. Quasi-simultaneous polarimetric data at $4300 \AA$ and $5250 \AA$. Each point represents data obtained within $63 \mathrm{~min}$ of each other. The straight lines show the 1:1 correspondence.

Table 1. Ellipses parameters in the $(Q, U)$ plane.

\begin{tabular}{lcc}
\hline \hline Parameter & $4300 \AA$ & $5250 \AA$ \\
\hline Ellipse center & $(0.04,-1.63)$ & $(0.26,-1.32)$ \\
Ratio of axes & 0.654 & 0.632 \\
Eccentricity & 0.75 & 0.77 \\
Rotation angle & $-67.7^{\circ}$ & $-83.3^{\circ}$ \\
\hline
\end{tabular}

variable accretion) and variable obscuration of the star by circumstellar matter. A few other sources of variability could perhaps explain RY Lup's behavior, such as pulsations and binarity. We discuss all these possibilities in turn.

\subsection{Possible mechanisms for the photometric and polarimetric variations}

Stellar pulsations cannot explain the data satisfactorily. If the pulsations were radial, i.e., spherically symmetric, then no changes in polarization would be expected. If the pulsations were non-radial, then many periods with differing amplitudes would be expected, leading to more complicated light and polarization variations than the observations show. Furthermore, $\mathrm{T}$ Tauri stars are located too far from the instability strip in the HR diagram, and the theory of pulsating variables (e.g. Cox 1980) predicts a period that is about $2 \mathrm{~h}$ shorter than the $3.75 \mathrm{~d}$ observed period. Gahm et al. (1989) also ruled out pulsations related to the deuterium-burning phase.

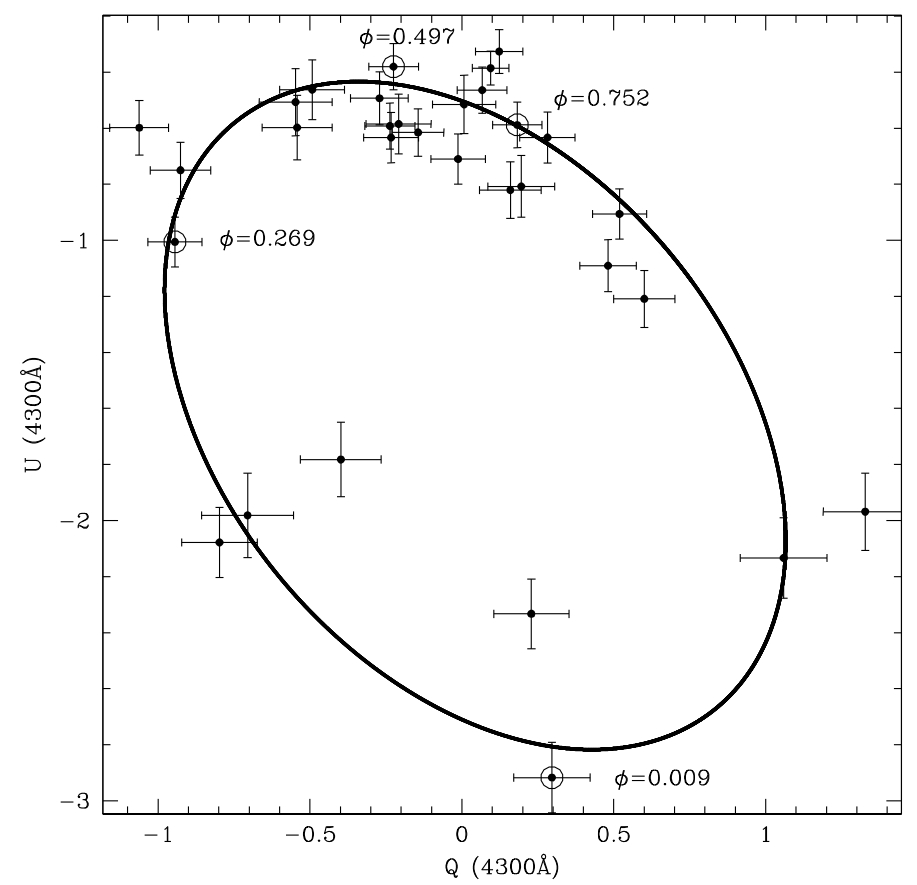

Fig. 9. Polarimetric data in the $Q U$ plane, at $4300 \AA$. The ellipse is a fit to the data. Four orbital phases are indicated by open circles.

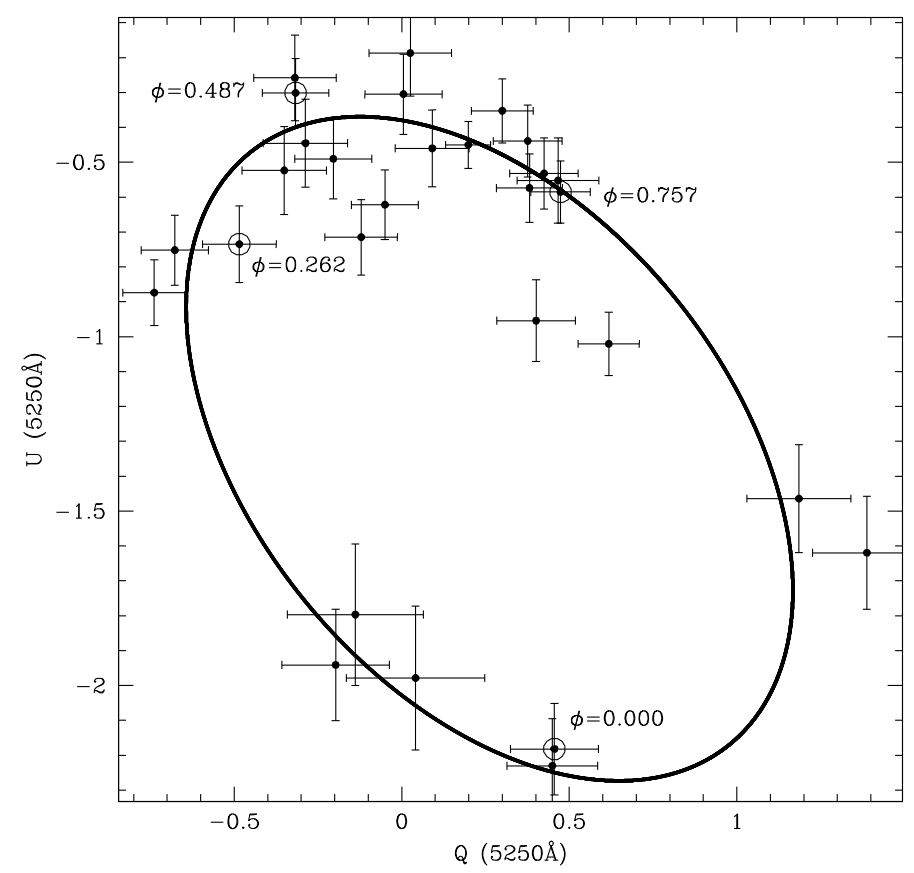

Fig. 10. Polarimetric data in the $Q U$ plane, at $5250 \AA$. The ellipse is a fit to the data. Four orbital phases are indicated by open circles.

There is currently no observational support for RY Lup being a binary system with a $3.75 \mathrm{~d}$ period. There is no evidence of a companion in the spectrum of RY Lup, and the phase of the light variations is not conserved rigorously. Even though RY Lup's polarization variations could be interpreted as that of a binary (Manset \& Bastien 2000, 2001), the observations cited in this paragraph do not appear to be compatible with this hypothesis.

The presence of large spots on the surface of rotating stars was proposed by Hoffmeister (1965) as a mechanism to explain the cyclic variations observed in stars like RY Lup. However, the presence of magnetic or accretion spots cannot explain the 


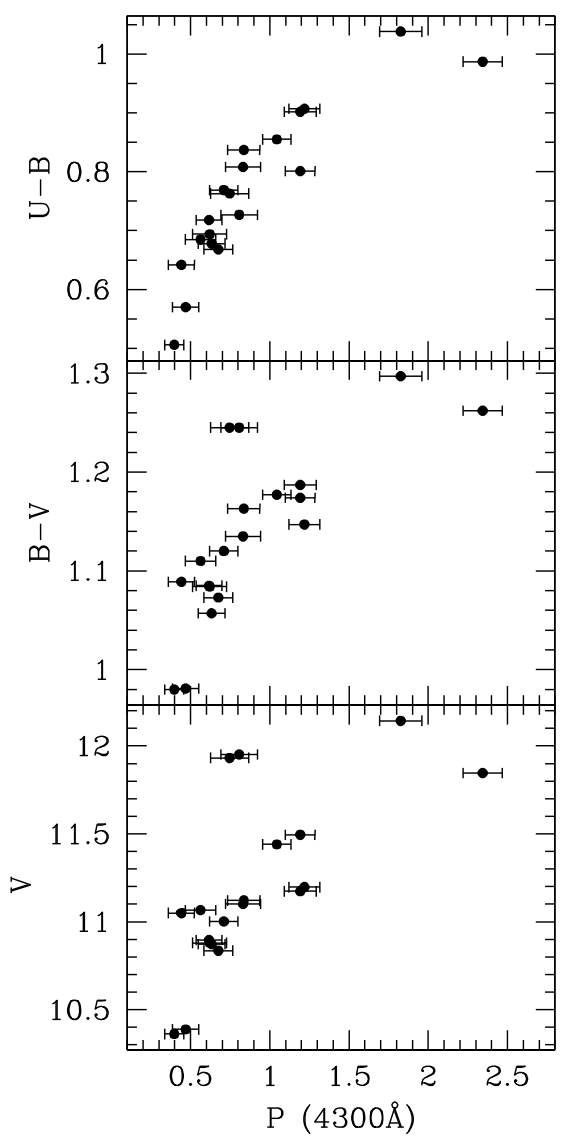

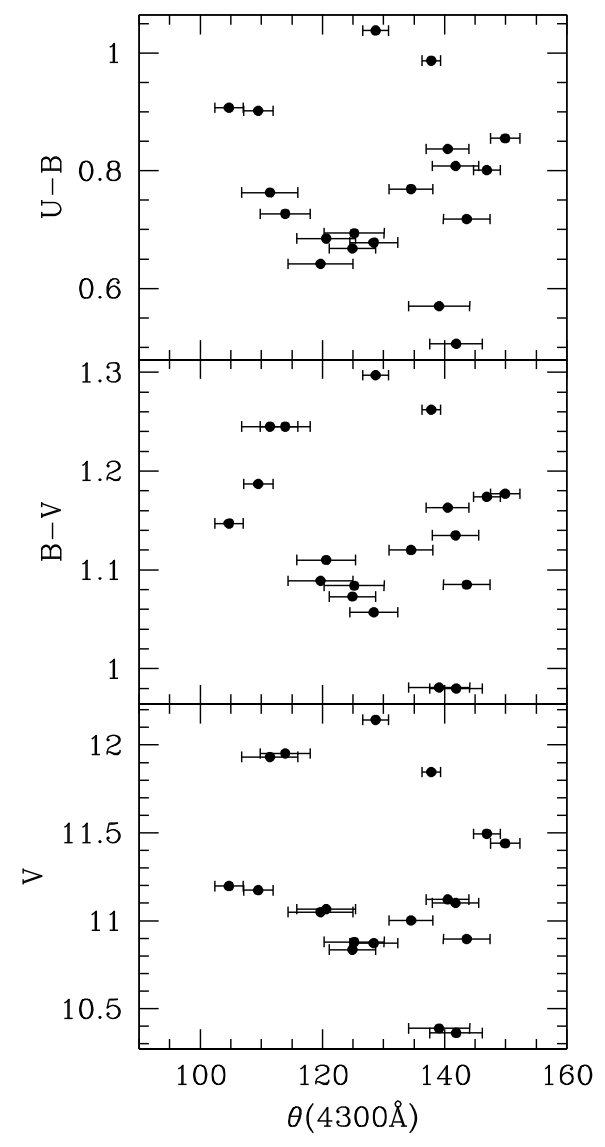

Fig. 11. Quasi-simultaneous photometry and polarimetry (intrinsic + interstellar) of RY Lup at $4300 \AA$ A. Each point represents data obtained within $29 \mathrm{~min}$ of one another. wavelength dependence of the observed photometric variations, nor does it explain the $\mathrm{H} \beta$ line variations and the infrared excess of RY Lup, a conclusion also reached by Liseau et al. (1987), Hutchinson et al. (1989), and Gahm et al. (1989). If magnetic or accretion spots were present on the stellar surface, they would affect the colors and spectral type, which are not observed to change when the star's brightness changes.

Bastien \& Landstreet (1979) have found that most of the polarization in TTSs arises in extended circumstellar dust envelopes outside the gas-emitting regions. The presence of dust around RY Lup is evident from the observed polarization and especially its variable wavelength dependence (Bastien 1985) and also from its near-infrared excess and its detection at farinfrared wavelengths by IRAS (Beichman et al. 1985). The existence of intrinsic polarization in RY Lup thus qualitatively agrees with the hypothesis of variable circumstellar extinction by dusty clouds orbiting the star. As far as the photometry is concerned, the reddening appears consistent with obscuration by interstellar grains with $R=3.1$ during the small amplitude variations. On the other hand, the deep minima (that did not occur during our observing run) seem to be associated with extinction by larger grains having $R \gtrsim 5.0$ (Evans et al.1982; Hutchinson et al. 1989).

Since the polarization position angle $\theta$ changes in time, a model with a circumstellar cloud passing right in front of the star (on the observer's line of sight) would not work; in that case, the position angle is not expected to change. According to Hutchinson et al. (1994), the relationship between the white light polarization and the $V$ magnitude is too steep compared to expectations (see Grinin et al. 1991) and cannot be explained by the occultation of an unpolarized source. Indeed, if dusty blobs orbiting around the star were responsible for the light and polarimetric variations, they would produce a polarization vector that describes a double loop in the $(Q, U)$ plane during one photometric period, as can be seen from the simple orbiting dust blob model by Bastien (1981). Such behavior is not compatible with our observations.

However, in the case of binary stars embedded in circumstellar dusty envelopes, Manset \& Bastien (2000, 2001) have found that several effects (eccentric orbits, variable absorption effects (pre- and post-scattering factors), the scattering functions of dust grains, and asymmetric envelopes) can cause single harmonic variations. Indeed, we see in Sect. 4.2 that RY Lup shows two peaks in polarization, with the one around phase 0.6 much weaker than the other one at phase 0.0 . Therefore, various effects could explain why RY Lup's polarimetric variations do not trace a double loop in the $(Q, U)$ plane.

It is however clear that a photopolarimetric model, such as the one developed for AA Tau (Bouvier et al. 1999; Ménard et al. 2003; O'Sullivan et al. 2005) with a warped disk resulting from the interaction between a disk and an inclined magnetosphere, explains the photometric and polarimetric observations of RY Lup quantitatively. The low-mass TTS AA Tau exhibits a roughly constant level of brightness, interrupted by quasi-periodic dips of over $1 \mathrm{mag}$, with an increase in polarization when the star is faint; i.e., it displays similar behavior to that of RY Lup.

The observed color variability of RY Lup (Sect. 3.2) can be explained easily by assuming that an accretion column and its accompanying accretion spot, both hot and not completely occulted by the disk, come in and out of view as proposed for AA Tau by Bouvier et al. (2003). We note in passing that Gahm et al. (1993b) proposed a similar idea for explaining the color variations of RY Lup. In the O'Sullivan et al. (2005) simulations, a Gaussian-shaped warp passing in front of AA Tau produces a 1 mag. drop in the photometry with a $0.4-0.6 \%$ increase 
Table 2. Coefficients of the 4 th order fit to the polarimetric data.

\begin{tabular}{ccc}
\hline \hline Coefficient & $4300 \AA$ & $5250 \AA$ \\
\hline$q_{0}$ & $-0.09522 \pm 0.00525$ & $+0.11603 \pm 0.00204$ \\
$q_{1}$ & $-0.59267 \pm 0.00708$ & $-0.47114 \pm 0.00264$ \\
$q_{2}$ & $+0.28937 \pm 0.00787$ & $+0.38391 \pm 0.00321$ \\
$q_{3}$ & $-0.11513 \pm 0.00811$ & $-0.05536 \pm 0.00323$ \\
$q_{4}$ & $+0.31425 \pm 0.00688$ & $+0.17599 \pm 0.00263$ \\
$q_{5}$ & $-0.15972 \pm 0.00805$ & $-0.14188 \pm 0.00308$ \\
$q_{6}$ & $+0.14853 \pm 0.00691$ & $+0.05233 \pm 0.00272$ \\
$q_{7}$ & $-0.12732 \pm 0.00696$ & $-0.12144 \pm 0.00266$ \\
$q_{8}$ & $-0.08816 \pm 0.00797$ & $-0.09157 \pm 0.00318$ \\
$u_{0}$ & $-1.15146 \pm 0.00527$ & $-1.01864 \pm 0.00203$ \\
$u_{1}$ & $-0.27516 \pm 0.00704$ & $-0.34508 \pm 0.00264$ \\
$u_{2}$ & $-0.93350 \pm 0.00789$ & $-0.80745 \pm 0.00322$ \\
$u_{3}$ & $-0.31211 \pm 0.00811$ & $-0.42408 \pm 0.00323$ \\
$u_{4}$ & $-0.30237 \pm 0.00688$ & $-0.22802 \pm 0.00263$ \\
$u_{5}$ & $+0.19206 \pm 0.00806$ & $+0.04816 \pm 0.00308$ \\
$u_{6}$ & $-0.01058 \pm 0.00691$ & $-0.01616 \pm 0.00272$ \\
$u_{7}$ & $+0.05492 \pm 0.00696$ & $-0.02673 \pm 0.00266$ \\
$u_{8}$ & $+0.00159 \pm 0.00797$ & $+0.01963 \pm 0.00318$ \\
\hline
\end{tabular}

in polarization, and the dip in photometry is slightly more pronounced in the blue than in the red. RY Lup dims by about $1 \mathrm{mag}$ in $V$ while its polarization increase by more than $2 \%$. For the warped disk to occult the star, the inclination of the disk must be high, and in the case of RY Lup, which displays more pronounced polarimetric variations, it should be higher than for AA Tau. We examine in the following whether a simple model aiming at reproducing the polarimetry only supports these assumptions.

\subsection{A polarization model}

It is customary when analyzing the polarization data of spectroscopic binaries to fit the data with a very general Fourier series, e.g., $Q=q_{0}+q_{1} \cos l+q_{2} \sin l+q_{3} \cos 2 l+q_{4} \sin 2 l+\ldots$

$U=u_{0}+u_{1} \cos l+u_{2} \sin l+u_{3} \cos 2 l+u_{4} \sin 2 l+\ldots$

where $l=2 \pi \phi$, with $\phi$ the orbital phase (see for example Manset \& Bastien 2002, 2003). Even for the case of RY Lup, which is not a binary, it is useful to adopt the same representation because it is quite general.

The results of the fourth order fit for RY Lup are given in Table 2 and plotted along with the polarization as a function of phase in Figs. 12 and 13. The coefficients of the Fourier fit higher than second order (in $2 l$ ) are not negligible. Including terms up to the fourth order reduces the normalized $\chi^{2}$ of the fit (see Table 3), especially at $4300 \AA$. We note the two peaks in polarization around phases 0.0 and 0.6 at both wavelengths.

For rotating astrophysical systems, Milgrom (1979) presented a formalism that allows one to compute the linear polarization produced by single Rayleigh scattering of unpolarized radiation in a system with arbitrary distributions of scatterers, absorbers, and radiation field. General relations between system properties and observable quantities can be derived. In particular, the inclination of the system can be obtained for systems in which the optical depth for absorption from any scatterer to infinity, along the line of sight, is independent of the phase (i.e., if absorption can be neglected).

Similar methods have been developed by Rudy \& Kemp (1978) and Brown et al. (1978, hereafter BME) in the case of binary stars. For the analysis and interpretation of polarization data from binaries, see Bastien (1988b) and Manset (2005).
Even though RY Lup is not a binary, its polarimetric variations are very similar to such systems. We therefore used both methods, Milgrom's and BME's, to estimate the inclination angle of the system. Both approaches only assume single scattering. This means that we are restricted to small grains $(\leqq 0.08 \mu \mathrm{m})$ distributed in an optically thin region $(\tau \leqq 0.1)$ around RY Lup. The assumption of single scattering is, however, over-simplistic, as it is not consistent with the achromatic extinction.

Milgrom's theory tells us that each set of four coefficients $q_{i}$ and $u_{i}$ for a given order in $l$ will yield an ellipse in the $(Q, U)$ plane. Figures 9 and 10 present the data in the Stokes parameters $(Q, U)$-plane, along with an ellipse fit to the data. The ellipse's parameters are given in Table 1. If one makes the additional assumption that the extinction from any scatterer to the observer is independent of phase, then the inclination of the rotation axis of the system can be determined independently for the first and second order terms in $l$ from the canonical relations given by Milgrom (1979).

The inclination found using both models, both wavelengths, and the first 2 orders are presented in Table 4. The value for the orbital inclination should not be a function of the wavelength of observation, but is different for the Milgrom method at $4300 \AA$, whereas the 3 other Milgrom values agree with each other and give an average of $85.6^{\circ} \pm 3^{\circ}$. The BME values for a given wavelength agree with one another, but the average inclination for the first order, $125.8^{\circ}$, differs from the value obtained from the second order, $95.9^{\circ}$. The difference between first and second order inclination values could stem from the fact that the system is not axially symmetric (e.g., a warp explains the data) and that first and second order coefficients may have different sensitivities to the non-axial density distribution. Since both orders contribute to the polarimetric variations, there is no reason to favor one value over the other. However, the Milgrom average value of $85.6^{\circ}$ points to an almost edge-on geometry, and suggests that the second order BME inclination, $95.9^{\circ}$, is correct. If the orientation of the inclination (i.e., if the rotation axis is pointing toward or away from the observer) is ambiguous, the values $85.6^{\circ}$ and $95.9^{\circ}$ are actually equivalent, and $\approx 5^{\circ}$ from an edge-on geometry.

We therefore conclude from our polarimetric observations that RY Lup's geometry is very close to edge-on. A model comprising a warped disk producing variable extinction because it is seen almost edge-on thus appears to be able to explain the data.

\subsection{Derived properties of RY Lup}

In the following we demonstrate that the period of 3.75 days found in the data is compatible with the rotation period of a G8 pre-main sequence star seen at a high inclination angle and derive the stellar parameters for RY Lup under this assumption. We conclude that the matter responsible for the periodic partial occultation of the star is corotating with the star.

To deduce the stellar parameters from the photometric and spectroscopic properties of RY Lup, we must compute its photospheric luminosity $L_{\text {star. }}$. Because both accretion and disk emission may contribute to the luminosity of young stellar objects, one must choose the photometric band that is least influenced by non-stellar emission to estimate the photospheric luminosity. Several methods for doing this have been proposed in the past. For example, Strom et al. (1989) normalized a blackbody with temperature equal to the stellar effective temperature $T_{\text {eff }}$ to the $R$-filter flux, while Kenyon \& Hartmann (1995) used the flux in the $J$-filter and a suitable bolometric correction to derive 


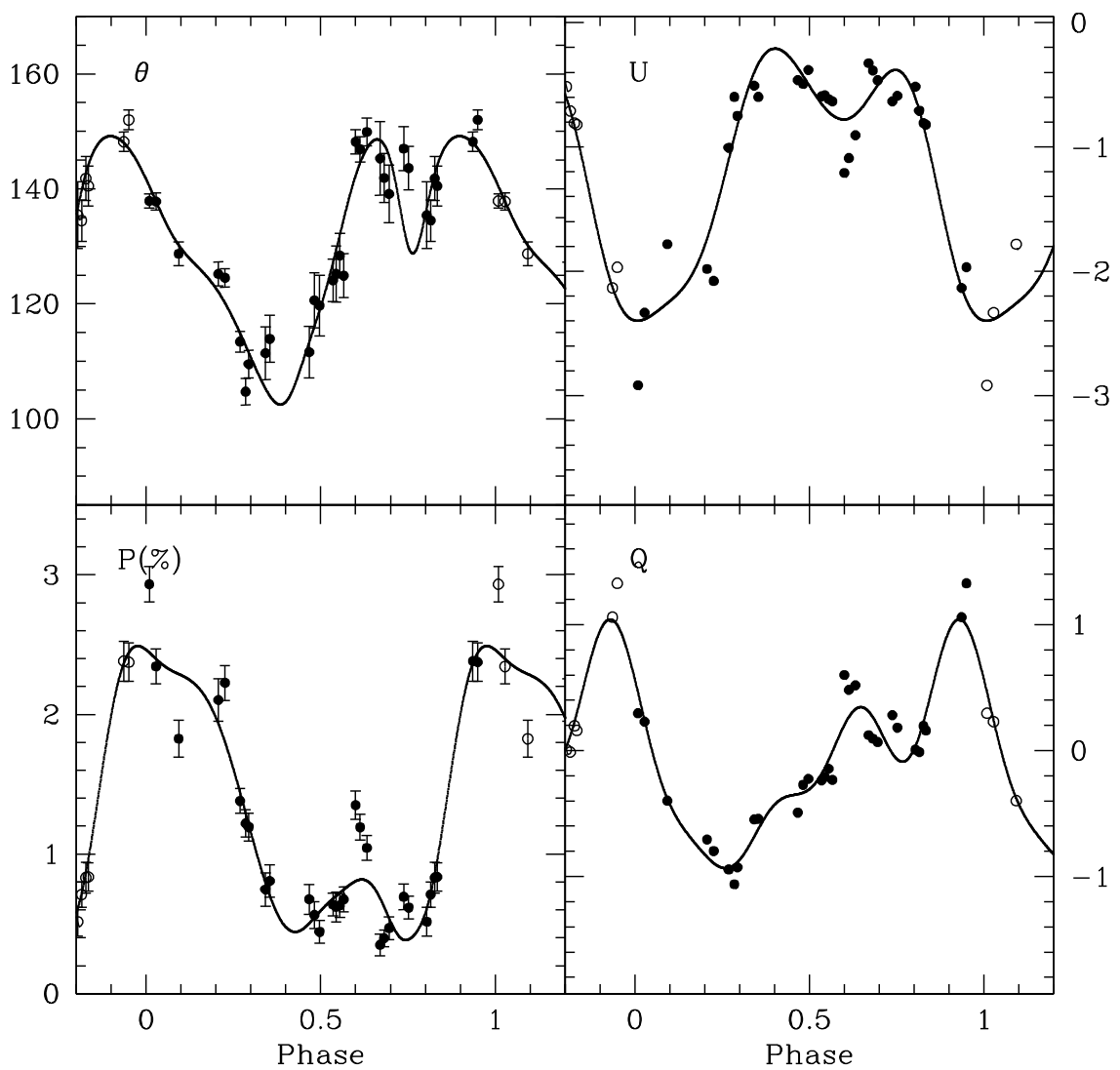

Fig. 12. Polarimetry of RY Lup at $4300 \AA$ as a function of phase, calculated with a period of $3.75 \mathrm{~d}$. The line represents the order 4 Fourier fit.

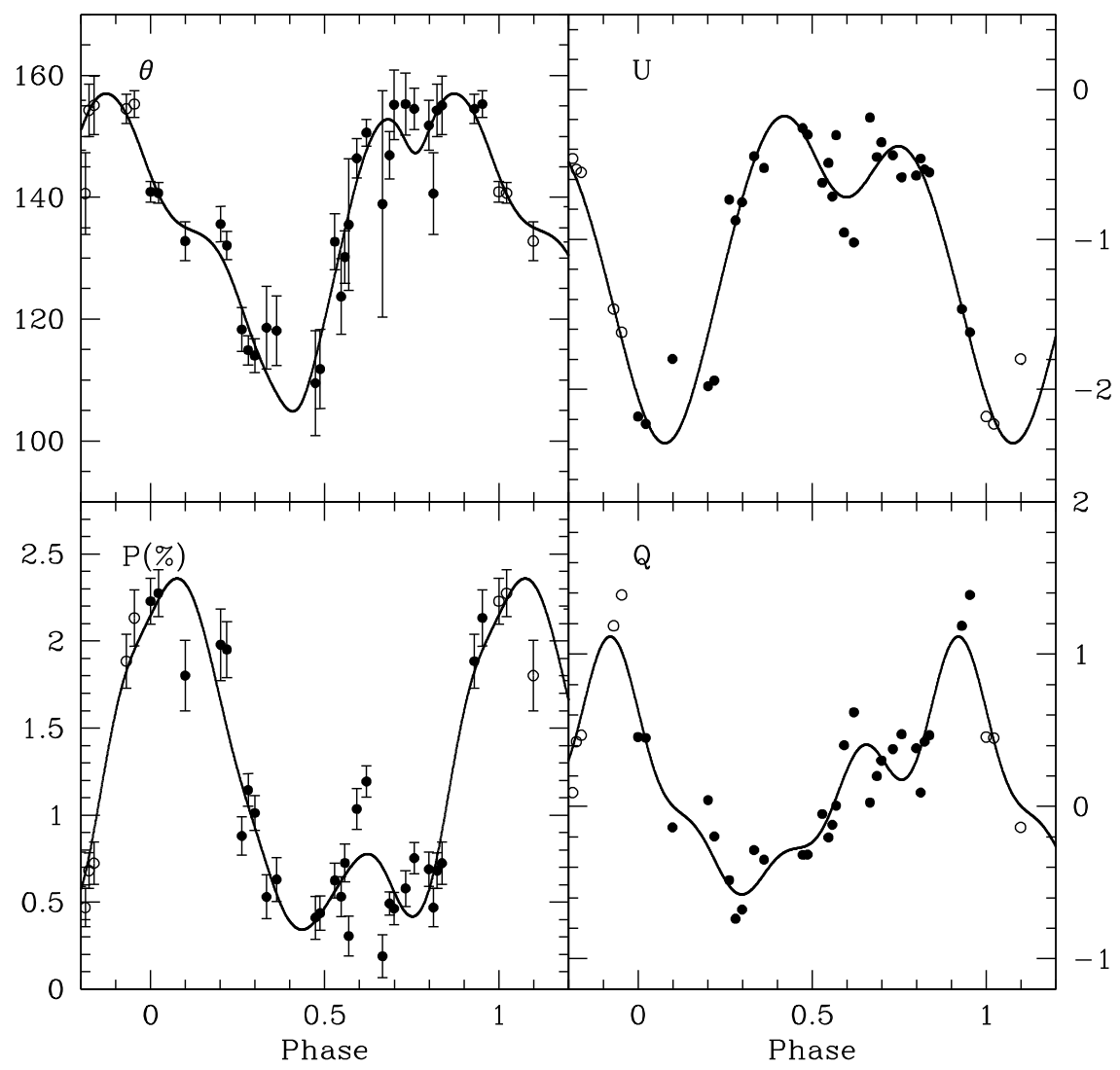

Fig. 13. Polarimetry of RY Lup at $5250 \AA$ as a function of phase, calculated with a period of $3.75 \mathrm{~d}$. The line represents the order 4 Fourier fit.

the photospheric luminosity of a large sample of Taurus stars. However, as Cieza et al. (2005) re-discovered recently ${ }^{3}$, the disk

3 The contribution of the disk to the $J$ flux of CTTSs had been noted in earlier works, e.g., Bertout et al. (1988). emission of accreting stars contributes significantly to the J-flux, so that the photospheric luminosities derived by Hartmann and Kenyon for CTTSs are systematically overestimated.

RY Lup displays a moderate UV excess and a sizable infrared excess, so it is plausible that part of its variability can be 
Table 3. $\chi^{2}$ values for the Fourier fit.

\begin{tabular}{ccc}
\hline \hline & Up to Order 2 & Up to Order 4 \\
\hline$\chi_{Q}^{2}$ at $4300 \AA$ & 7.18 & 4.17 \\
$\chi_{U}^{2}$ at $4300 \AA$ & 7.04 & 6.85 \\
$\chi_{Q}^{2}$ at $5250 \AA$ & 3.09 & 3.77 \\
$\chi_{U}^{2}$ at $5250 \AA$ & 5.06 & 4.05 \\
\hline
\end{tabular}

Table 4. Orbital inclinations found using the Milgrom and BME models.

\begin{tabular}{lccc}
\hline \hline Wavelength & Model & First Order & Second Order \\
\hline $4300 \AA$ & Milgrom & 53.2 & 83.7 \\
& BME & $126.8 \pm 1.7$ & $96.3 \pm 1.2$ \\
$5250 \AA$ & Milgrom & 88.6 & 84.5 \\
& BME & $124.8 \pm 2.1$ & $95.5 \pm 1.4$ \\
\hline
\end{tabular}

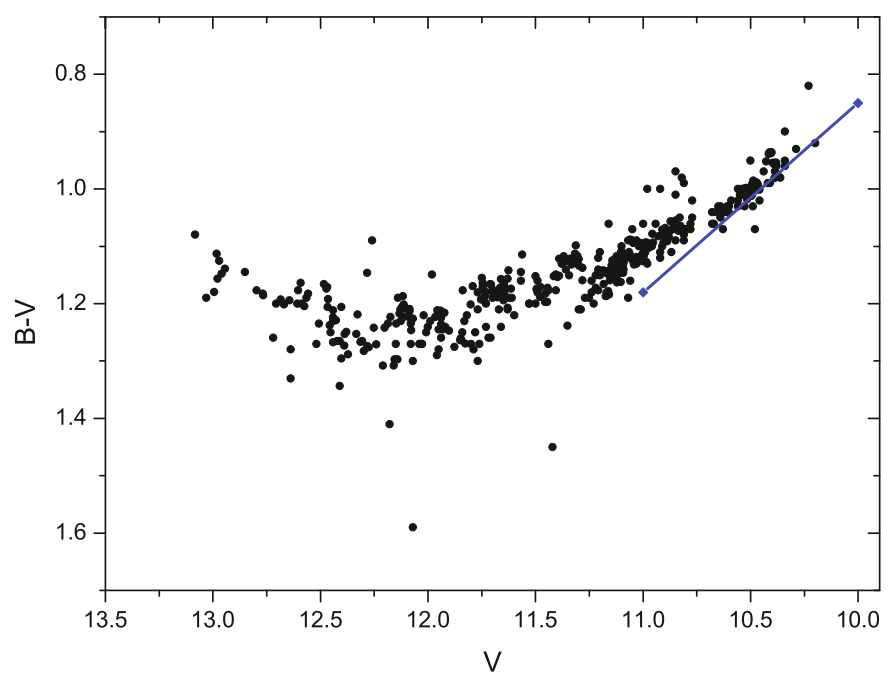

Fig. 14. Correlation between $B$ and $B-V$ as derived from the full RY Lupi data set (filled circles). The solid line shows how a star moves in the diagram when it suffers a 1 magnitude extinction change.

attributed to accretion. As recommended by Cieza et al. (2005) we therefore use the observed flux in I and the relevant bolometric correction here (using the standard values given by Kenyon \& Hartmann 1995; for a G8 star) to derive the photospheric luminosity of RY Lup. An additional complication stems from the wide variability range of RY Lup, since we must determine the magnitude within this range that corresponds to the emission from the clean photosphere.

We note here that the two main mechanisms that can be invoked for explaining the light variability of RY Lup, i.e., disk accretion and the occultation of the central star, act in opposite directions; accretion increases the $V$-luminosity, while occultation due to dust clouds decreases it. Also, the $B$ and $V$ fluxes are correlated in accretion events (e.g., Bertout et al. 1988), while we expect some de-correlation of these fluxes in occultation events, due to the combined effects of absorption (resulting in a $B-V$ increase) and scattering (which produces a decrease in $B-V$ ). As illustrated in Fig. 14, the RY Lupi $V$ vs. $B-V$ scatter diagram displays a correlation between $B$ and $B-V$ when the star is brightest, while the scatter increases and the star's color becomes bluer when it becomes faint. One might be tempted to attribute the $V$ variations around maximum light $(10.3 \leq V \leq 10.7)$ to variable accretion, but one notices that they occur along the direction expected from a change in extinction. It thus appears that the full
Table 5. Properties of RY Lupi derived assuming two different distances and their associated uncertainties.

\begin{tabular}{ccccc}
\hline \hline Distance $(\mathrm{pc})$ & $L_{\text {star }} / L_{\odot}$ & $M_{\text {star }} / M_{\odot}$ & $R_{\text {star }} / R_{\odot}$ & Age $(\mathrm{Myr})$ \\
\hline 189 & 4.3 & 1.65 & 2.15 & 8.2 \\
147 & 2.6 & 1.38 & 1.67 & 13.3 \\
120 & 1.7 & 1.19 & 1.35 & 20.0 \\
\hline 156 & 2.9 & 1.43 & 1.78 & 11.8 \\
108 & 1.4 & - & - & - \\
83 & 0.8 & - & - & - \\
\hline
\end{tabular}

Note. A dash means that no position on the pre-main sequence evolutionary track for a G8 star is compatible with this combination of data.

variability of RY Lupi in $B$ and $V$ is likely caused by changes in the extinction caused by the disk matter and that its photospheric brightness corresponds to the largest observed flux in the optical filters. That there are no obvious manifestations of accretion in the optical photometry of RY Lupi can probably be attributed to the low contrast between photospheric emission and accretion spot emission that is expected for $\mathrm{G}$ and earlier spectral types.

We recall in passing that, as seen in Sect. 3, the probability of observing RY Lupi in the $V$ range $11.0-11.5$ is 0.36 and the probability of observing the star in a brighter (dimmer) state is 0.23 (0.41). From Fig. 14, and from the extinction of RY Lupi at maximum light being $\approx 0.5 \mathrm{mag}$ (see below), we conclude that the extinction in front of the star is lower than 1.5 magnitude during about $59 \%$ of the observing time or, equivalently, during the same fraction of the rotation period.

Coming back to the photospheric flux, we use the average value of the brightest $V$ measurements as a representative estimate. We take $V=10.34, V-I=1.08$. From there, and assuming a spectral type G8, we find $A_{\mathrm{V}}=0.47$. We then derive photospheric luminosities from the $I$-flux, assuming two different distances $147_{-27}^{+42} \mathrm{pc}$ (Bertout et al. 1999) and $108_{-25}^{+48} \mathrm{pc}$ (Perryman et al. 1997) and deduce masses, stellar radii, and ages of RY Lupi for the average and extrema distance values from the Siess et al. (2000) evolutionary tracks. These results are summarized in Table 5. As seen there, the nominal parallax value of RY Lupi as given in the Hipparcos catalog is incompatible with a position on the pre-main sequence track of a G8 star; the minimum distance leading to a luminosity compatible with that track is $120 \mathrm{pc}$.

Since we now have an estimated range of values for the stellar radius, we can use the relation

$v \sin i \cdot P_{\mathrm{rot}}=2 \pi R_{\mathrm{star}} \sin i$

to derive the stellar inclination angle. Using the values $v \sin i=$ $25.0 \pm 4.6 \mathrm{~km} \mathrm{~s}^{-1}$ (Bouvier et al. 1986) and $P_{\text {rot }}=3.75 \mathrm{~d}$, we can rewrite this equation as a condition on $R_{\mathrm{star}}$ :

$\frac{R_{\text {star }}}{R_{\odot}} \geq 1.84 \pm .0 .34$,

where the error bars reflect the uncertainty on $v \sin i$. Obviously, given these uncertainties we cannot constrain the star's inclination angle precisely but are able to conclude that all the information available on RY Lupi is consistent with a system comprising a G8 star surrounded by an edge-on disk, and we can derive a range of stellar masses and ages compatible with the derived parallax and stellar radius values. We find that the mass of RY Lupi is $M_{\text {star }} / M_{\odot}=1.71 \pm 0.43$, while its age is $(1.2 \pm 0.4) \times 10^{7} \mathrm{yr}$.

It has been argued in the previous sections that stellar spots cannot be responsible for the observed simultaneous photometric and polarimetric variations and that the matter responsible for 
Table 6. Photometric data for RY Lup.

\begin{tabular}{|c|c|c|c|c|c|c|c|c|c|}
\hline $\begin{array}{c}\text { Date } \\
\text { JD-2 } 400000.0\end{array}$ & $\overline{\bar{V}}$ & $B-V$ & $\overline{U-B}$ & Phase & $\begin{array}{c}\text { Date } \\
\text { JD-2 } 400000.0\end{array}$ & $\overline{\bar{V}}$ & $B-V$ & $\overline{U-B}$ & Phase \\
\hline 45013.801 & 10.362 & 0.980 & 0.506 & 0.680 & 45020.826 & 10.878 & 1.074 & 0.653 & 0.553 \\
\hline 45013.834 & 10.372 & 0.977 & 0.597 & 0.689 & 45020.832 & 10.873 & 1.057 & 0.678 & 0.555 \\
\hline 45013.869 & 10.388 & 0.981 & 0.570 & 0.698 & 45020.857 & 10.859 & 1.066 & 0.672 & 0.561 \\
\hline 45013.871 & 10.387 & 0.970 & 0.581 & 0.698 & 45020.863 & 10.824 & 1.064 & 0.656 & 0.563 \\
\hline 45016.810 & 11.066 & 1.110 & 0.685 & 0.482 & 45020.877 & 10.836 & 1.073 & 0.668 & 0.567 \\
\hline 45016.818 & 11.070 & 1.090 & 0.683 & 0.484 & 45020.882 & 10.848 & 1.055 & 0.679 & 0.568 \\
\hline 45016.822 & 11.063 & 1.114 & 0.664 & 0.485 & 45021.795 & 10.995 & 1.113 & 0.779 & 0.811 \\
\hline 45016.857 & 11.047 & 1.094 & 0.654 & 0.495 & 45021.801 & 11.002 & 1.120 & 0.769 & 0.813 \\
\hline 45016.868 & 11.050 & 1.089 & 0.642 & 0.498 & 45021.862 & 11.101 & 1.135 & 0.808 & 0.829 \\
\hline 45016.885 & 11.060 & 1.088 & 0.690 & 0.502 & 45021.868 & 11.106 & 1.131 & 0.830 & 0.831 \\
\hline 45017.816 & 10.903 & 1.081 & 0.698 & 0.750 & 45021.880 & 11.121 & 1.163 & 0.837 & 0.834 \\
\hline 45017.825 & 10.897 & 1.085 & 0.718 & 0.753 & 45021.885 & 11.125 & 1.159 & 0.847 & 0.835 \\
\hline 45017.830 & 10.886 & 1.091 & 0.719 & 0.754 & 45022.865 & 12.143 & 1.297 & 1.038 & 0.097 \\
\hline 45017.855 & 10.890 & 1.083 & 0.728 & 0.761 & 45022.873 & 12.154 & 1.297 & 1.018 & 0.099 \\
\hline 45017.863 & 10.896 & 1.086 & 0.690 & 0.763 & 45022.888 & 12.161 & 1.308 & 1.178 & 0.103 \\
\hline 45017.881 & 10.898 & 1.068 & 0.727 & 0.768 & 45023.776 & 11.932 & 1.245 & 0.763 & 0.340 \\
\hline 45017.888 & 10.905 & 1.093 & 0.726 & 0.770 & 45023.784 & 11.938 & 1.225 & 0.755 & 0.342 \\
\hline 45018.804 & 11.902 & 1.247 & 1.062 & 0.014 & 45023.802 & 11.966 & 1.223 & 0.760 & 0.347 \\
\hline 45018.814 & 11.878 & 1.275 & 0.934 & 0.017 & 45023.810 & 11.952 & 1.245 & 0.727 & 0.349 \\
\hline 45018.839 & 11.849 & 1.261 & 0.972 & 0.023 & 45023.863 & 11.956 & 1.211 & 0.796 & 0.363 \\
\hline 45018.847 & 11.846 & 1.262 & 0.987 & 0.025 & 45023.870 & 11.940 & 1.236 & 0.752 & 0.365 \\
\hline 45018.868 & 11.850 & 1.263 & 1.009 & 0.031 & 45023.882 & 11.942 & 1.212 & 0.764 & 0.368 \\
\hline 45018.875 & 11.829 & 1.269 & 1.027 & 0.033 & 45023.888 & 11.922 & 1.216 & 0.807 & 0.370 \\
\hline 45019.797 & 11.210 & 1.177 & 0.884 & 0.279 & 45024.791 & 11.496 & 1.175 & 0.819 & 0.610 \\
\hline 45019.805 & 11.219 & 1.172 & 0.903 & 0.281 & 45024.797 & 11.495 & 1.174 & 0.801 & 0.612 \\
\hline 45019.839 & 11.197 & 1.147 & 0.907 & 0.290 & 45024.814 & 11.479 & 1.188 & 0.809 & 0.617 \\
\hline 45019.847 & 11.183 & 1.166 & 0.921 & 0.292 & 45024.821 & 11.478 & 1.171 & 0.776 & 0.618 \\
\hline 45019.860 & 11.174 & 1.187 & 0.902 & 0.296 & 45024.836 & 11.457 & 1.197 & 0.796 & 0.622 \\
\hline 45019.867 & 11.171 & 1.186 & 0.904 & 0.297 & 45024.859 & 11.446 & 1.197 & 0.788 & 0.629 \\
\hline 45019.883 & 11.157 & 1.184 & 0.892 & 0.302 & 45024.866 & 11.454 & 1.182 & 0.763 & 0.630 \\
\hline 45019.889 & 11.163 & 1.178 & 0.979 & 0.303 & 45024.878 & 11.440 & 1.177 & 0.855 & 0.634 \\
\hline 45020.802 & 10.880 & 1.084 & 0.694 & 0.547 & 45024.884 & 11.443 & 1.172 & 0.796 & 0.635 \\
\hline 45020.808 & 10.882 & 1.074 & 0.670 & 0.548 & $\ldots$ & $\ldots$ & $\ldots$ & $\ldots$ & $\ldots$ \\
\hline
\end{tabular}

these variations is very likely associated with the disk itself. For the stellar mass given above, the Keplerian radius for a period of 3.75 days is $\approx 6.5 R_{\text {star. }}$. We therefore have two possible scenarios. The disk material that is responsible for occulting the star could be located at the Keplerian radius by chance (since there is no obvious physical reason it should be located precisely there) or it could be corotating with the star because it is located at the disk edge and trapped by the stellar magnetosphere, in analogy with the case of AA Tau. With no information on the magnetic-field strength of RY Lupi, it is impossible to derive a precise magnetospheric radius, but a range of a few stellar radii is usually assumed for typical CTTSs with kGauss magnetic field strengths, so that this second possibility appears plausible.

\section{Conclusions}

RY Lup is a T Tauri star with a $60 \%$ chance of being observed in a state brighter than $V=11.5 \mathrm{mag}$. Our re-analysis of 622 photometric measurements obtained in the $V$ band from the late 1970 s to 1990 also shows that there is less than a $10 \%$ chance of observing RY Lupi within 0.5 magnitude of its brightest state in $V$, and the star is fainter than $V=12.5$ about $10 \%$ of the time.

From our $B V$ polarimetric and $U B V$ photometric data obtained simultaneously during 12 consecutive nights, we conclude the following.

- There are large amplitude photometric variations $\Delta V=$ $1.2 \mathrm{mag}, \Delta(B-V)=0.25 \mathrm{mag}$, and $\Delta(U-B)=0.37 \mathrm{mag}$.
- There are large amplitude variations for the observed polarization at $4300 \AA, \Delta P=2.3 \%, \Delta \theta=48^{\circ}$, and at $5250 \AA$, $1.8 \%$, and $47^{\circ}$.

- When RY Lup is bright, a lower polarization is observed; and when the polarization is high, the star is fainter and also redder.

- A period search on all our data yielded a period of $3.74_{-0.18}^{+0.20}$ days.

- After subtracting an estimate of the IS polarization, we find that RY Lup's intrinsic polarization is high: $1.30 \%$ at $130^{\circ}$ at $4300 \AA$, and $1.22 \%$ at $133^{\circ}$ at $5250 \AA$.

- Polarimetric variations have a slightly higher amplitude in the blue at $4300 \AA$ than in the green at $5250 \AA$. The observed position angle varies by $\approx 45^{\circ}$ at both wavelengths.

- The polarization and position angle at $4300 \AA$ are correlated with the polarization and position angle at $5250 \AA$.

Based on the position angle of the polarization, and taking the interstellar polarization into account, we predict that the circumstellar disk is oriented at $\approx 135^{\circ}$ if optically thick, or at $\approx 45^{\circ}$ if optically thin. The photometric and polarimetric variations can be explained by variable occultation of the stellar light caused by an almost edge-on (within $\approx 5^{\circ}$ ) circumstellar disk that is warped close to the star, where is interacts with the star's magnetosphere.

Acknowledgements. We are grateful to R. Lamontagne, A. F. Moffat, and L. Drissen for useful discussions. Special thanks go to G. Hutchinson for making his data available before publication and for sharing his enthusiasm on this star. 
Table 7. Polarimetric data for RY Lup at $4300 \AA$.

\begin{tabular}{cccccc}
\hline \hline Date & $P$ & $\begin{array}{c}\sigma_{P} \\
\text { JD-2 400 000.0 }\end{array}$ & $\begin{array}{c}\theta \\
{ }^{\circ}\end{array}$ & $\begin{array}{c}\sigma_{\theta} \\
{ }^{\circ}\end{array}$ & Phase \\
\hline 45013.764 & 0.35 & 0.08 & 145.3 & 6.4 & 0.670 \\
45013.808 & 0.40 & 0.06 & 141.9 & 4.3 & 0.682 \\
45013.861 & 0.47 & 0.08 & 139.1 & 5.0 & 0.696 \\
45014.762 & 2.38 & 0.14 & 148.2 & 1.7 & 0.936 \\
45014.814 & 2.37 & 0.14 & 152.0 & 1.7 & 0.950 \\
45015.778 & 2.10 & 0.15 & 125.2 & 2.1 & 0.207 \\
45015.850 & 2.23 & 0.12 & 124.5 & 1.6 & 0.226 \\
45016.754 & 0.68 & 0.11 & 111.6 & 4.5 & 0.467 \\
45016.809 & 0.56 & 0.09 & 120.6 & 4.8 & 0.482 \\
45016.867 & 0.44 & 0.08 & 119.7 & 5.3 & 0.497 \\
45017.769 & 0.69 & 0.09 & 147.0 & 3.8 & 0.738 \\
45017.821 & 0.62 & 0.08 & 143.6 & 3.8 & 0.752 \\
45018.785 & 2.93 & 0.13 & 137.9 & 1.2 & 0.009 \\
45018.856 & 2.34 & 0.12 & 137.8 & 1.5 & 0.028 \\
45019.761 & 1.38 & 0.09 & 113.4 & 1.8 & 0.269 \\
45019.821 & 1.22 & 0.10 & 104.7 & 2.3 & 0.285 \\
45019.853 & 1.19 & 0.10 & 109.5 & 2.4 & 0.294 \\
45020.757 & 0.64 & 0.08 & 124.1 & 3.7 & 0.535 \\
45020.792 & 0.62 & 0.11 & 125.2 & 4.9 & 0.544 \\
45020.833 & 0.63 & 0.08 & 128.4 & 3.9 & 0.555 \\
45020.875 & 0.67 & 0.09 & 124.9 & 3.8 & 0.566 \\
45021.766 & 0.51 & 0.10 & 135.4 & 5.8 & 0.804 \\
45021.810 & 0.71 & 0.09 & 134.5 & 3.6 & 0.815 \\
45021.852 & 0.83 & 0.11 & 141.8 & 3.8 & 0.827 \\
45021.879 & 0.84 & 0.10 & 140.5 & 3.5 & 0.834 \\
45022.850 & 1.83 & 0.13 & 128.7 & 2.1 & 0.093 \\
45023.783 & 0.75 & 0.12 & 111.4 & 4.6 & 0.342 \\
45023.831 & 0.81 & 0.11 & 113.9 & 4.1 & 0.354 \\
45024.752 & 1.35 & 0.10 & 148.2 & 2.1 & 0.600 \\
45024.800 & 1.19 & 0.09 & 146.9 & 2.2 & 0.613 \\
45024.872 & 1.04 & 0.09 & 149.9 & 2.4 & 0.632 \\
\hline & & & & &
\end{tabular}

Table 8. Polarimetric data for RY Lup at $5250 \AA$.

\begin{tabular}{cccccc}
\hline \hline Date & $\begin{array}{c}P \\
\text { JD-2 400 000.0 }\end{array}$ & $\begin{array}{c}\sigma_{P} \\
\%\end{array}$ & $\begin{array}{c}\theta \\
{ }^{\circ}\end{array}$ & $\begin{array}{c}\sigma_{\theta} \\
{ }^{\circ}\end{array}$ & Phase \\
\hline 45013.748 & 0.19 & 0.12 & 138.9 & 18.6 & 0.666 \\
45013.826 & 0.49 & 0.07 & 146.9 & 3.9 & 0.686 \\
45013.872 & 0.46 & 0.09 & 155.2 & 5.7 & 0.699 \\
45014.738 & 1.88 & 0.15 & 154.5 & 2.4 & 0.930 \\
45014.825 & 2.13 & 0.16 & 155.3 & 2.2 & 0.953 \\
45015.754 & 1.99 & 0.21 & 135.6 & 2.9 & 0.201 \\
45015.822 & 1.95 & 0.16 & 132.1 & 2.3 & 0.219 \\
45016.774 & 0.41 & 0.12 & 109.5 & 8.6 & 0.473 \\
45016.828 & 0.44 & 0.10 & 111.8 & 6.5 & 0.487 \\
45017.748 & 0.58 & 0.10 & 155.3 & 5.1 & 0.732 \\
45017.841 & 0.75 & 0.09 & 154.5 & 3.4 & 0.757 \\
45018.753 & 2.23 & 0.13 & 140.9 & 1.7 & 0.000 \\
45018.834 & 2.27 & 0.13 & 140.7 & 1.7 & 0.022 \\
45019.736 & 0.88 & 0.11 & 118.3 & 3.6 & 0.262 \\
45019.801 & 1.14 & 0.09 & 114.9 & 2.4 & 0.280 \\
45019.872 & 1.01 & 0.10 & 114.0 & 2.8 & 0.299 \\
45020.737 & 0.62 & 0.10 & 132.7 & 4.6 & 0.529 \\
45020.804 & 0.53 & 0.11 & 123.7 & 6.2 & 0.547 \\
45020.845 & 0.72 & 0.11 & 130.2 & 4.3 & 0.558 \\
45020.887 & 0.30 & 0.11 & 135.5 & 10.8 & 0.569 \\
45021.749 & 0.69 & 0.10 & 151.8 & 4.1 & 0.799 \\
45021.796 & 0.47 & 0.11 & 140.6 & 6.7 & 0.812 \\
45021.839 & 0.68 & 0.10 & 154.3 & 4.3 & 0.823 \\
45021.891 & 0.72 & 0.12 & 155.1 & 4.8 & 0.837 \\
45022.875 & 1.80 & 0.20 & 132.8 & 3.2 & 0.099 \\
45023.749 & 0.53 & 0.13 & 118.6 & 6.8 & 0.333 \\
45023.860 & 0.63 & 0.13 & 118.1 & 5.7 & 0.362 \\
45024.722 & 1.03 & 0.12 & 146.4 & 3.2 & 0.592 \\
45024.827 & 1.19 & 0.09 & 150.6 & 2.2 & 0.620 \\
\hline & & & & &
\end{tabular}

This work was supported by the Sonderforschungsbereih 131-Radioastronomie and by the Natural Sciences and Engineering Research Counil of Canada.

\section{References}

Alves, F. O., \& Franco, G. A. P. 2006, MNRAS, 366, 238

Appenzeller, I., Jankovics, I., \& Krautter, J. 1983, A\&AS, 53, 291

Baisch, S., \& Bokelmann, G. H. R. 1999, Computers \& Geosciences, 25, 739

Bastien, P. 1981, A\&A, 94, 294

Bastien, P. 1982, A\&AS, 48, 153, 513

Bastien, P. 1985, ApJS, 59, 277

Bastien, P. 1988a, in Polarized radiation of circumstellar origin, ed. G. V. Coyne, A. F. J. Moffat, S. Tapia, A. M. Magalhães, R. E. SchulteLadbeck, \& D. T. Wickramasinghe (Vatican City State/Tucson: AZ Vatican Observatory/University of Arizona Press), 541

Bastien, P. 1988b, in Polarized radiation of circumstellar origin, ed. G. V. Coyne, A. F. J. Moffat, S. Tapia, A. M. Magalhães, R. E. SchulteLadbeck, \& D. T. Wickramasinghe (Vatican City State/Tucson: AZ Vatican Observatory/University of Arizona Press), 595

Bastien, P., \& Landstreet, J. 1979, ApJ, 229, L137

Beichmann, C. A., Neugebauer, G., Habing, H. J., et al. 1985, IRAS Catalogs and Atlases

Bertout, C., Basri, G., \& Bouvier, J. 1988, ApJ, 330, 350

Bertout, C., Robichon, N., \& Arenou, F. 1999, A\&A, 352, 574

Boesgaard, A. M. 1984, AJ, 89, 1635

Bouvier, J., Bertout, C., \& Bouchet, P. 1986, A\&A, 158, 149

Bouvier, J., Bertout, C., \& Bouchet, P. 1988, A\&AS 75, 1

Bouvier, J., Chelli, A., Allain, S. et al. 1999, A\&A, 349, 619

Bouvier, J, Grankin, K. N., Alencar, S. H. P., et al. 2003, A\&A, 409, 169

Brown, J. C., McLean, I. S., \& Emslie, A. 1978, A\&A, 68, 415

Cieza, L. A., Kessler-Silacci, J. E., Jaffe, D. T., et al. 2005, ApJ, 635, 422

Comeron, F., in Handbook of Star Forming Regions, ASP Conf. Ser., in preparation

Covino, E., Terranegra, L., Franchini, M., et al. 1992, A\&AS, 94, 273

Cox, J. P. 1980, Theory of Stellar Pulsations (Princeton: Princeton University Press), 10

Drissen, L., Lamontagne, R., Moffat, A. F. J., et al. 1986, ApJ, 304, 188

Drissen, L., Bastien, P., \& St-Louis, N. 1989, AJ, 97, 814

Evans, A., Bode, M. F., Whittet, D. C.B, et al. 1982, MNRAS, 199, 37

Franco, G. A. P. 2002, MNRAS, 331, 474

Gahm, G. F., Fischerström, C., Liseau, R., et al. 1989, A\&A, 211, 115

Gahm, G. F., Gullbring, E., Fischerström, et al. 1993a, A\&AS, 100, 371

Gahm, G. F., Liseau, R., Gullbring, E., et al. 1993b, A\&A, 279, 477

Glass, I. S., \& Penston, M. V. 1974, MNRAS, 167, 237

Grinin, V. P., Kiselev, N. N., Chernova, G. P., et al. 1991, Ap\&SS, 186, 283

Heiles, C. 2000, AJ, 119, 923

Herbig, G. H. 1977, ApJ, 214, 747

Herbig, G. H., \& Kameswara Rao, N. 1972, ApJ, 174, 401

Herbst, W., Herbst, D. K., Grossman, E. J., \& Weinstein, D. 1994, AJ, 108, 1906

Hoffmeister, C. 1949, Astron. Nachr., 278, 24

Hoffmeister, C. 1958, Veröff. Sternw. Sonneberg, 3, 340

Hoffmeister, C. 1965, Veröff. Sonneberg, 6, 97

Hughes, J., Hartigan, P., Krautter, J., et al. 1994, AJ, 108, 1071

Hutchinson, M. G., Evans, A., Davies, J., et al. 1987, n Circumstellar Matter, ed. I. Appenzeller, \& C. Hordan (Dordrecht: Reidel), Proc. IAU Symp., 122, 109

Hutchinson, M. G., Evans, A., Davies, J. K., et al. 1989, MNRAS, 237, 683

Hutchinson, M. G., Albinson, J. S., Barrett, P., et al. 1994, A\&A, 285, 883

Isella, A., \& Natta, A. 2005, A\&A, 438, 899

Kenyon, S. J., \& Hartmann, L. 1995, ApJS, 101, 117

Liseau, R., Lindroos, K. P., \& Fischerström, C. 1987, A\&A, 183, 274

Manset, N. 2005, in Polarimetry of Binary Stars, Astronomical Polarimetry Current Status and Future Directions, ASP Conf. Ser. 343, 389

Manset, N., \& Bastien, P. 2000, AJ, 120, 413

Manset, N., \& Bastien, P. 2001, AJ, 122, 2692

Manset, N., \& Bastien, P. 2002, AJ, 124, 1089

Manset, N., \& Bastien, P. 2003, AJ, 125, 3274

Ménard, F., \& Bertout, C. 1999, in The Origin of Stars and Planetary Systems, NATO ASIC Proc., 540, 341

Ménard, F., Bouvier, J., Dougados, C., et al. 2003, A\&A, 409, 163

Milgrom, M. 1979, A\&A, 76, 338

O'Sullivan, M., Truss, M., Walker, C., et al. 2005, MNRAS, 358, 632

Perryman, M. A. C., Lindegren, L., Kovalevsky, J., et al. 1997, A\&A, 323, 49

Rudy, R. J., \& Kemp, J. C. 1976, ApJ, 207, L125

Serkowski, K., Mathewson, D. L., \& Ford, V. L. 1975, ApJ, 196, 261

Siess, L., Dufour, E., \& Forestini, M. 2000, A\&A, 358, 593

Strom, K. M., Strom, S. E., Edwards, S., et al. 1989, AJ, 97, 1451

Yudin, R. V., \& Evans, A. 1998, A\&AS, 131, 401 